\title{
Bleeding disorders in adolescents with heavy menstrual bleeding in a multicenter prospective US cohort
}

\author{
Ayesha Zia, ${ }^{1,2,3,4}$ Shilpa Jain, ${ }^{5}$ Peter Kouides, ${ }^{6}$ Song Zhang, ${ }^{1,7}$ Ang Gao, ${ }^{1,7}$ \\ Niavana Salas, ${ }^{4}$ May Lau, ${ }^{1,3,8}$ Ellen Wilson,,${ }^{1,4,9}$ Nicole DeSimone $^{1,10}$ \\ and Ravi Sarode $\mathrm{I}^{1,10}$ \\ ${ }^{1}$ The University of Texas Southwestern Medical Center, Dallas, TX; ${ }^{2}$ Department of \\ Pediatrics, Children's Medical Center, Dallas, TX; 'Division of Hematology/Oncology, \\ Children's Medical Center, Dallas, TX; ${ }^{4}$ Children's Medical Center, Dallas, TX; ${ }^{5} \mathrm{Hemophilia}$ \\ Center of Western New York, John R. Oishei Children's Hospital of Buffalo, Division of \\ Pediatric Hematology-Oncology, Buffalo, NJ; ${ }^{6}$ Mary M. Gooley Hemophilia Center, \\ University of Rochester School of Medicine, Rochester, MN; 'Department of Data and \\ Population Sciences, The University of Texas Southwestern Medical Center, Dallas, TX; \\ ${ }^{8}$ Division of Adolescent Medicine, The University of Texas Southwestern Medical Center, \\ Dallas, TX; ${ }^{\circ}$ Department of Obstetrics and Gynecology, The University of Texas \\ Southwestern Medical Center, Dallas, TX and ${ }^{10}$ Department of Pathology and Internal \\ Medicine, The University of Texas Southwestern Medical Center, Dallas, TX, USA
}

\section{ABSTRACT}

$\mathrm{H}$ eavy menstrual bleeding is common in adolescents. The frequency and predictors of bleeding disorders in adolescents, especially with anovulatory bleeding, are unknown. Adolescents referred for heavy menstrual bleeding underwent an evaluation of menstrual bleeding patterns, and bleeding disorders determined a priori. The primary outcome was the diagnosis of a bleeding disorder. Two groups were compared: anovulatory and ovulatory bleeding. Multivariable logistic regression analysis of baseline characteristics and predictors was performed. Kaplan Meier curves were constructed for the time from the first bleed to bleeding disorder diagnosis. In 200 adolescents, a bleeding disorder was diagnosed in 33\% $(n=67)$ : low von Willebrand factor levels in 16\%, von Willebrand disease in $11 \%$, and qualitative platelet dysfunction in $4.5 \%$. The prevalence of bleeding disorder was similar between ovulatory and anovulatory groups ( $31 \%$ vs. $36 \% ; P=0.45)$. Predictors of bleeding disorder included: younger age at first bleed (OR: 0.83; 95\%CI: 0.73, 0.96), Hispanic ethnicity (OR: 2.48; 95\%CI: $1.13,5.05)$, non-presentation to emergency department for heavy bleeding (OR: 0.14; 95\%CI: 0.05, 0.38), and International Society on Thrombosis and Haemostasis (ISTH) Bleeding Assessment Tool score $\geq 4$ (OR: 8.27; 95\%CI: $2.60,26.44)$. Time from onset of the first bleed to diagnosis was two years in the anovulatory, and six years in the ovulatory cohort (log-rank test, $P<0.001)$. There is a high prevalence of bleeding disorders in adolescents with heavy periods, irrespective of the bleeding pattern. Among bleeding disorders, the prevalence of qualitative platelet dysfunction is lower than previously reported.

\section{Introduction}

Heavy menstrual bleeding (HMB) is common in adolescents after menarche. ${ }^{1}$ Anovulation is the most common etiology of $\mathrm{HMB}$ and is expected to persist for up to five years. ${ }^{2}$ Underlying bleeding disorders (BD) are another important etiology of $\mathrm{HMB}$ affecting up to $20 \%$ of adults and $13-60 \%$ of adolescents. ${ }^{3}$ A large body of research has focused on the prevalence of $\mathrm{BD}$ in adult women and its predictors. In contrast, pediatric literature on $\mathrm{BD}$ frequency has only been recently accrued. Most data are retrospective and prevalence studies by and large excluded women with anovulatory bleeding. ${ }^{4 \cdot 6}$ Data on predictors of $\mathrm{BD}$ in adolescents with $\mathrm{HMB}$, when anovulation is most prevalent, are scant and it is difficult to determine whom to screen for $\mathrm{BD} .^{3}$
Haematologica 2020
Volume 105(7):1969-1976

\section{Correspondence:}

AYESHA ZIA

Ayesha.zia@utsouthwestern.edu

Received: April 29, 2019.

Accepted: October 14, 2019.

Pre-published: October 17, 2019.

doi:10.3324/haematol.2019.225656

Check the online version for the most updated information on this article, online supplements, and information on authorship \& disclosures: www.haematologica.org/content/105/7/1969

\section{(C)2020 Ferrata Storti Foundation}

Material published in Haematologica is covered by copyright. All rights are reserved to the Ferrata Storti Foundation. Use of published material is allowed under the following terms and conditions:

https://creativecommons.org/licenses/by-nc/4.0/legalcode. Copies of published material are allowed for personal or internal use. Sharing published material for non-commercial purposes is subject to the following conditions:

https://creativecommons.org/licenses/by-nc/4.0/legalcode, sect. 3. Reproducing and sharing published material for commercial purposes is not allowed without permission in writing from the publisher. 
Our study aimed to describe the frequency, predictors, and time from bleeding onset to $\mathrm{BD}$ diagnosis in a prospective cohort of adolescents with $\mathrm{HMB}$, using a welldefined and rigorous protocol of hemostasis testing and platelet function analysis. We hypothesized that the frequency of undiagnosed $\mathrm{BD}$ in adolescents with $\mathrm{HMB}$ would be similar to adults, and that the menstrual bleeding pattern will not predict a BD.

\section{Methods}

Postmenarchal adolescents up to 18 years of age referred for $\mathrm{HMB}$ without a diagnosis of $\mathrm{BD}$ were eligible to participate (Figure 1). Participants were enrolled prospectively from the Young Women's Blood Disorders Program at The University of Texas Southwestern (UTSW), Dallas, TX (primary site), USA, and the Children's Hospital of Buffalo (CHOB), NY, USA (external site) between July 2014 and December 2017, after informed consent and/or assent. ${ }^{7}$ The institutional review board at both UTSW and CHOB (Institutional Review Board numbers: STU 102014-001 and 00003126, respectively) approved the study. Participants were excluded if they did not complete: 1) a minimum of two visits and 2) the minimum $\mathrm{BD}$ evaluation, decided a priori.

\section{Definitions}

Heavy menstrual bleeding was defined by menstrual duration $\geq 7$ days with a sensation of "gushing" or "flooding" or bleeding through a pad or tampon for $\leq 2$ hours. ${ }^{8,9}$ Based on the pattern of $\mathrm{HMB}$ in the entire cohort, participants were classified and compared among two groups: the anovulatory $\mathrm{HMB}$ group was defined as having menstrual duration $<21$ or $>45$ days; the ovulatory $\mathrm{HMB}$ group was defined when this menstrual pattern was not present. $^{10}$

\section{Laboratory testing}

All participants underwent testing that included complete blood count, serum ferritin, prothrombin time (PT), activated partial thromboplastin time (aPTT), fibrinogen, von Willebrand Factor (vWF) analysis (repeated at least twice for every participant), platelet aggregation (repeated if abnormal), Factor (F) XI, FXIII factor assays and systemic hyperfibrinolysis assessment using rotational thromboelastometry (ROTEM). ${ }^{11}$ This comprised the minimum $\mathrm{BD}$ evaluation for retention into the final analysis.

\section{Iron deficiency/iron deficiency anemia}

We defined anemia as hemoglobin $<110 \mathrm{~g} / \mathrm{L}$ and iron deficiency as serum ferritin levels $\leq 20 \mu \mathrm{g} / \mathrm{L}$.

\section{Bleeding assessment tools}

Pictorial Blood Assessment Chart (PBAC) - a PBAC score was com- puted for all participants, and an average score of three cycles was taken as the final score. ${ }^{12}$ Standardized pads and tampons were not provided.

\section{Outcomes}

The primary outcome of the study was the diagnosis of $\mathrm{BD}$ defined as low von Willebrand disease (vWD), qualitative platelet dysfunction (OPD), clotting factor deficiencies or hyperfibrinolysis.

\section{Statistical analysis}

Sample size - based on adult data, ${ }^{4,5}$ we assumed that $20 \%$ of adolescents with $\mathrm{HMB}$, irrespective of menstrual bleeding pattern, will be diagnosed with $\mathrm{BD}$ within six months from study entry; with a total of 180 patients, we estimated the rate of $\mathrm{BD}$ in this group with such precision that the $95 \%$ confidence interval has a half-length smaller than $8 \% .^{14}$

\section{Main outcome}

The frequency of BD was compared among groups using $\chi^{2}$ test.

A multivariable logistic regression model was built following the technique described by Hosmer, Lemeshow, and Sturdivant. ${ }^{15}$ Variable selection was performed using the stepwise procedure.

Kaplan-Meier curves were constructed from the first bleed to $\mathrm{BD}$ diagnosis for the entire cohort and the main two groups and compared using the log-rank test.

Further information on the methods used is available in the Online Supplementary Appendix.

\section{Results}

\section{Demographic and clinical characteristics}

Two hundred and thirty-five consecutive adolescents were enrolled during the study period, of which 200 participants met eligibility criteria, and had complete outcome data: 185 from UTSW and 15 from CHOB. The median age of participants was 15 years (y) (Table 1), and $28 \%$ were Hispanic. Fifty-five percent had normal body mass index (BMI) as determined by Center for Disease Control (CDC) growth charts. The median age at menarche was 12 years, and $\mathrm{BD}$ evaluation occurred at a median of three years from menarche. Thirty-three percent presented to the Emergency Department (ED) for evaluation and management of $\mathrm{HMB} ; 25 \%$ needed to be hospitalized, of which $19 \%$ received packed red blood cells (pRBC). The mean PBAC score was 386 [standard deviation (SD), 243] and the ISTH score was 3.5 (SD 1.3). A

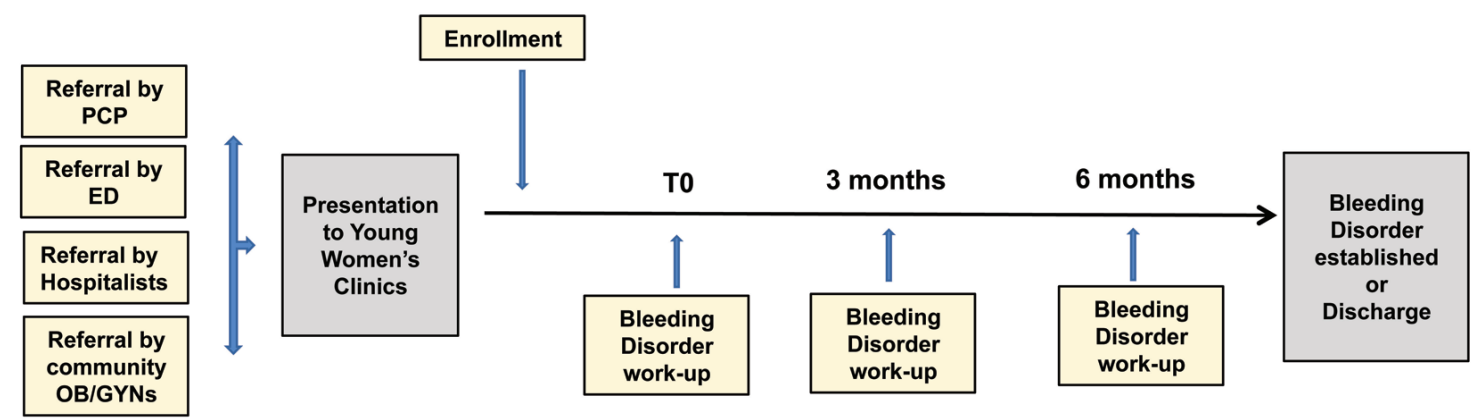

Figure 1. Study design. Prospective, multicenter, observational study of adolescents presenting with heavy menstrual bleeding. 
family history of bleeding symptoms or established BD was present in $21 \%$ and $6.5 \%$, respectively. Family history of gynecologic or obstetric bleeding was present in $60 \%$ : $57 \%$ ( $n=114)$ with HMB alone; $1.5 \%(n=3)$ with $\mathrm{HMB}$ and post-partum hemorrhage $(\mathrm{PPH})(\mathrm{n}=3)$, and $1.5 \%$ $(n=3)$ had only PPH. Of the first-degree relatives with $\mathrm{HMB}, 38 \%$ underwent hysterectomy for $\mathrm{HMB}(\mathrm{n}=44)$ and $2 \%$ underwent endometrial ablation $(n=2)$ to control $\mathrm{HMB}$. Thirty-six percent $(\mathrm{n}=72)$ had iron deficiency anemia, 29\% ( $n=57)$ had iron deficiency alone, and overall iron deficiency was documented in $65 \% \quad(n=129)$. Participants with anovulatory $\mathrm{HMB}$ had a higher BMI $(66.7 \%$ vs. $36 \%)$ and increased frequency of refractory $\mathrm{HMB}(34 \%$ vs. 19\%), ED visits (42\% vs. $25 \%$ ), hospitalizations ( $28 \%$ vs. $17 \%)$, and pRBC (24\% vs. $14 \%)$ admin- istration for $\mathrm{HMB}$ compared to those with ovulatory pattern bleeding.

\section{Frequency and types of bleeding disorders and additional bleeding symptoms}

Overall, 33\% ( $n=67)$ of adolescents were diagnosed with a $\mathrm{BD}$. Among those with $\mathrm{BD}$, there were no differences in the frequency of $\mathrm{BD}$ in the anovulatory and the ovulatory $\mathrm{HMB}$ groups ( $31 \%$ vs. $36 \% ; P=0.45)$. Low vWF levels were detected in $57 \%$ ( $n=38$ of 67$) ; 25 \%$ ( $n=17$ of 67) were diagnosed with vWD (type $1=13$, type $2=4$ ), $13.5 \%$ ( $n=9$ of 67 ) with QPD, and two participants were found to have coagulation factor deficiencies (one with mild FVIII deficiency (FVIII:C= 29\%) and mild FXIII deficiency (FXIII: 40\%). One participant, referred for $\mathrm{HMB}$

Table 1. Baseline characteristics of the entire study cohort and according to menstrual bleeding pattern.

\begin{tabular}{|c|c|c|c|}
\hline & $\begin{array}{c}\text { All patients } \\
(\mathrm{N}=200) \\
n(\%)\end{array}$ & $\begin{array}{c}\text { Anovulatory HMB } \\
\begin{array}{c}(\mathbf{N}=\mathbf{1 0 0}) \\
n(\%)\end{array}\end{array}$ & $\begin{array}{c}\text { Ovulatory HMB } \\
\begin{array}{c}(\mathrm{N}=100) \\
n(\%)\end{array}\end{array}$ \\
\hline Age, $y$ & $15(10-19)$ & $14(10-18)$ & $15(11-19)$ \\
\hline \multicolumn{4}{|l|}{ Ethnicity } \\
\hline Hispanic & $56(28)$ & $33(33)$ & $23(23)$ \\
\hline Non-Hispanic & $144(72)$ & $67(67)$ & $77(77)$ \\
\hline \multicolumn{4}{|l|}{ Race } \\
\hline White & $131(65)$ & $70(70)$ & $61(61)$ \\
\hline Black & $41(20.5)$ & $13(13)$ & $28(28)$ \\
\hline Other & $28(14)$ & $17(17)$ & $11(11)$ \\
\hline BMI, kg/m² & $23 \cdot 3(14 \cdot 5-55)$ & $24 \cdot 4(17-55)$ & $22.9(14 \cdot 5-44)$ \\
\hline Normal weight & $110(55)$ & $45(45)$ & $65(65)$ \\
\hline Overweight/Obese & $90(45)$ & $55(55)$ & $35(35)$ \\
\hline Age at menarche, $y$ & $12(9-15)$ & $12(9-15)$ & $12(9-15)$ \\
\hline Years since menarche & $3(1-4)$ & $3(1-4)$ & $3(1-4)$ \\
\hline Time to HMB, y & $0.5(0-5)$ & $0.5(0-5)$ & $0.4(0-5)$ \\
\hline Age at first bleed & $12(2-17)$ & $12(2-16)$ & $12(2-17)$ \\
\hline Refractory $\mathrm{HMB}^{\$}$ & $53(26 \cdot 5)$ & $34(34)$ & $19(19)$ \\
\hline Presentation to ED for $\mathrm{HMB}^{@}$ & $67(33.5)$ & $42(42)$ & $25(25)$ \\
\hline$\leq 1$ visit & $47(23.5)$ & $26(26)$ & $21(21)$ \\
\hline$\geq 2$ visits & $19(9 \cdot 5)$ & $15(15)$ & $4(4)$ \\
\hline Hospitalizations for HMB & $45(22.5)$ & $28(28)$ & $17(17)$ \\
\hline $\mathrm{pRBC}$ & $38(84)$ & $24(86)$ & $14(82)$ \\
\hline Parenteral Iron & $76(38)$ & $36(36)$ & $40(40)$ \\
\hline PBAC at study entry*\# & $386(243)$ & $427(274)$ & $345(201)$ \\
\hline ISTH-BAT* & $3.5(1 \cdot 3)$ & $3.4(1 \cdot 3)$ & $3.5(1 \cdot 3)$ \\
\hline FMH of bleeding or BD & $55(27.5)$ & $28(28)$ & $27(27)$ \\
\hline FMH of gynecologic or obstetric bleeding & $120(60)$ & $55(55)$ & $65(65)$ \\
\hline Hypermobility & $20(20)$ & $9(23)$ & 11(17) \\
\hline Hemoglobin, g/L & $11.8(3.4-16.4)$ & $11 \cdot 7(3 \cdot 9-15)$ & $11.8(3.4-16 \cdot 4)$ \\
\hline Ferritin, $\mathrm{ng} / \mathrm{mL}$ & $8(0.9-78)$ & $10.2(0.9-70)$ & $7 \cdot 2(1-98)$ \\
\hline TSH, mIU/L & $1.5(1.2-8.4)$ & $1.7(0.5-8.4)$ & $1.4(1 \cdot 2-5 \cdot 1)$ \\
\hline BD diagnosis & $67(33)$ & $31(31)$ & $36(36)$ \\
\hline
\end{tabular}

Values are medians with interquartile ranges except normally distributed variables marked with an asterisk; mean and standard deviations are reported. BMI: body mass index; ED: emergency department; HMB: heavy menstrual bleeding; ISTH-BAT: International Society of Thrombosis Haemostasis-Bleeding Assessment Tool; pRBC: packed red blood cells; PBAC: pictorial blood assessment chart;TSH: thyroid-stimulating hormone; y: years. Statistically significant differences between the anovulatory and ovulatory groups were found for refractory HMB, presentation to ED and PBAC @ \# $\$ P=0.01$. 
Table 2. Types of bleeding disorders in the whole group and according to menstrual bleeding pattern.

\begin{tabular}{|c|c|c|c|}
\hline & $\begin{array}{c}\text { All patients } \\
\begin{array}{c}(N=200) \\
n(\%)\end{array}\end{array}$ & $\begin{array}{c}\text { Anovulatory HMB } \\
\begin{array}{c}(\mathrm{N}=100) \\
n(\%)\end{array}\end{array}$ & $\begin{array}{c}\text { Ovulatory HMB } \\
\begin{array}{c}(\mathrm{N}=100) \\
\text { n }(\%)\end{array}\end{array}$ \\
\hline Low & $38(19)$ & $14(14)$ & $24(24)$ \\
\hline vWF & $17(8.5)$ & $12(12)$ & $5(5)$ \\
\hline Type 1 & $13(6.5)$ & $5(5)$ & $8(8)$ \\
\hline Type 2 & $4(2)$ & $3(3)$ & $1(1)$ \\
\hline Qualitative platelet dysfunction & $9(2)$ & $5(5)$ & $4(4)$ \\
\hline Clotting factor deficiencies & $2(1.5)$ & $2(2)$ & - \\
\hline Symptomatic hemophilia carrier & $1(0.5)$ & $1(1)$ & - \\
\hline
\end{tabular}

vWF: von Willebrand factor; vWD: von Willebrand disease.

A

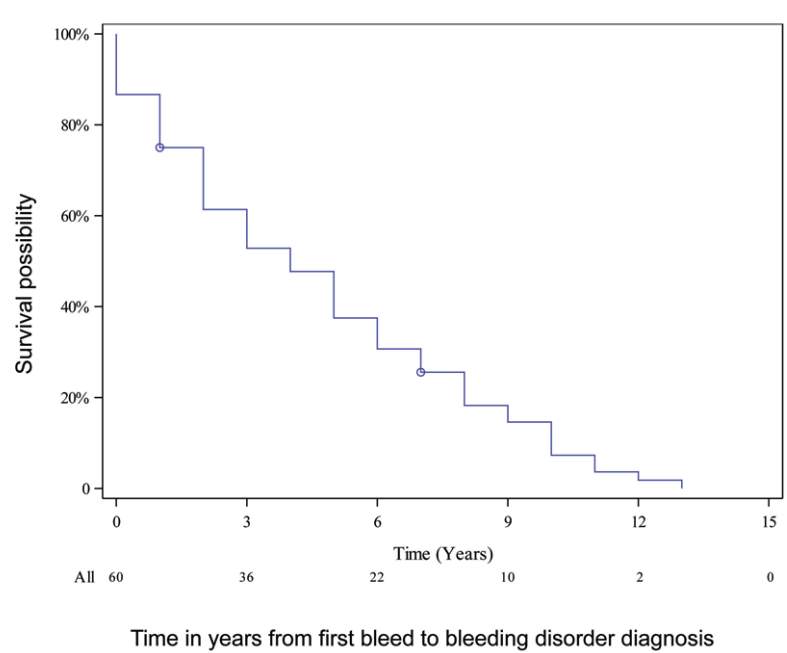

B

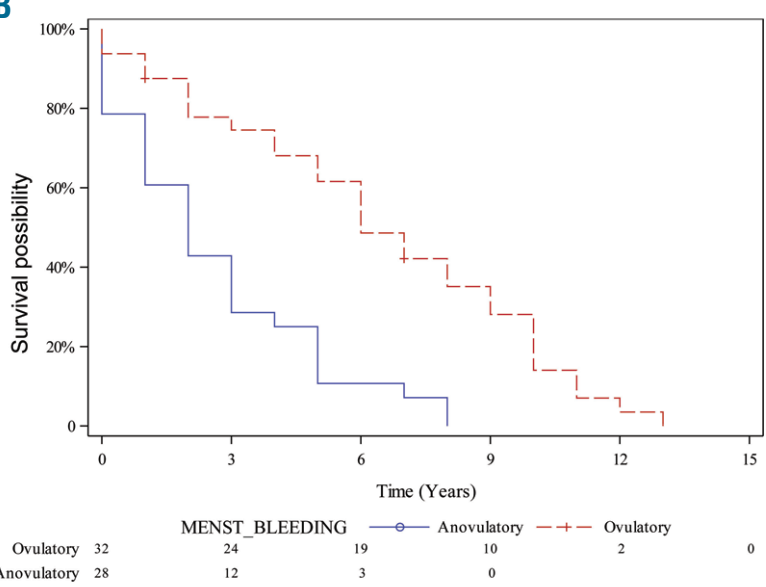

Time in years from first bleed to bleeding disorder diagnosis according to group

Figure 2. Kaplan-Meier curves showing time from the first bleeding event to bleeding disorder diagnosis. (A) Time until diagnosis for the entire group. (B) Time according to the menstrual bleeding group. The number of patients diagnosed according to the group is shown at the bottom of the graphic, on the x-axis.

four years after menarche because of a brother with FVIII deficiency, was diagnosed to be a symptomatic hemophilia carrier (FVIII: 124\%; positive for a pathogenic type 1 intron 22 inversion mutation (F8 c.6429+?_6430-? inv.). None of the participants showed evidence of systemic hyperfibrinolysis based on our testing protocol, though specific testing for plasminogen activator inhibitor or antiplasmin deficiency were not performed (Table 2 and Online Supplementary Tables S3 and S4). Of those diagnosed with BD, 29 (43\%) had $\mathrm{HMB}$ as the sole complaint, $21(31 \%)$ had one additional, $12(18 \%)$ had two, four $(6 \%)$ had three, and one participant had five additional bleeding symptoms. Of those without BD, 118 (88\%) had $\mathrm{HMB}$ as the sole complaint; $12(9 \%)$ had one, and $3(2 \%)$ had two additional bleeding symptoms in addition to $\mathrm{HMB}$ (Online Supplementary Table S5).

\section{Predictors of bleeding disorders}

The results of the univariable logistic regression of potential predictors of BD are shown in Table 3 . The final model (multivariable, stepwise logistic regression) included four predictors: younger age at first bleeding event, Hispanic ethnicity, non-presentation to ED, and ISTH BAT score of $\geq 4$.

\section{Time to diagnosis}

Kaplan-Meier curves showed that the median time from the first bleed to $\mathrm{BD}$ diagnosis was four years (range 2-5) in the entire cohort, two years (range 1-3) in the anovulatory group, and six years (range 4-9) in the ovulatory group. There was a significant difference in time to diagnosis across groups (log-rank test, $P<0.0001$ ) (Figure 2).

\section{Concomitant non-hemostatic disorders}

Twenty-three participants with $\mathrm{BD}$ were diagnosed with additional disorders: three $(1.5 \%)$ with polycystic ovarian syndrome, and four with uterine structural abnormalities (three with endometriosis and one with uterine polyps). Four participants without BD were diagnosed with vWF exon 28 polymorphism p.D1472H (suspected based on isolated decreased vWF:RCo) (Table 4). Only 100 participants underwent a joint exam for benign joint hypermobility $(\mathrm{BJH})$; of those, 20 met criteria for $\mathrm{BJH}$.

\section{Discussion}

Our study systematically investigated adolescents with $\mathrm{HMB}$ and showed that $33 \%$ had a BD. Almost $80 \%$ of 
Table 3. Univariate and multivariate analyses of the association between baseline patient characteristics and bleeding disorder in the entire cohort.

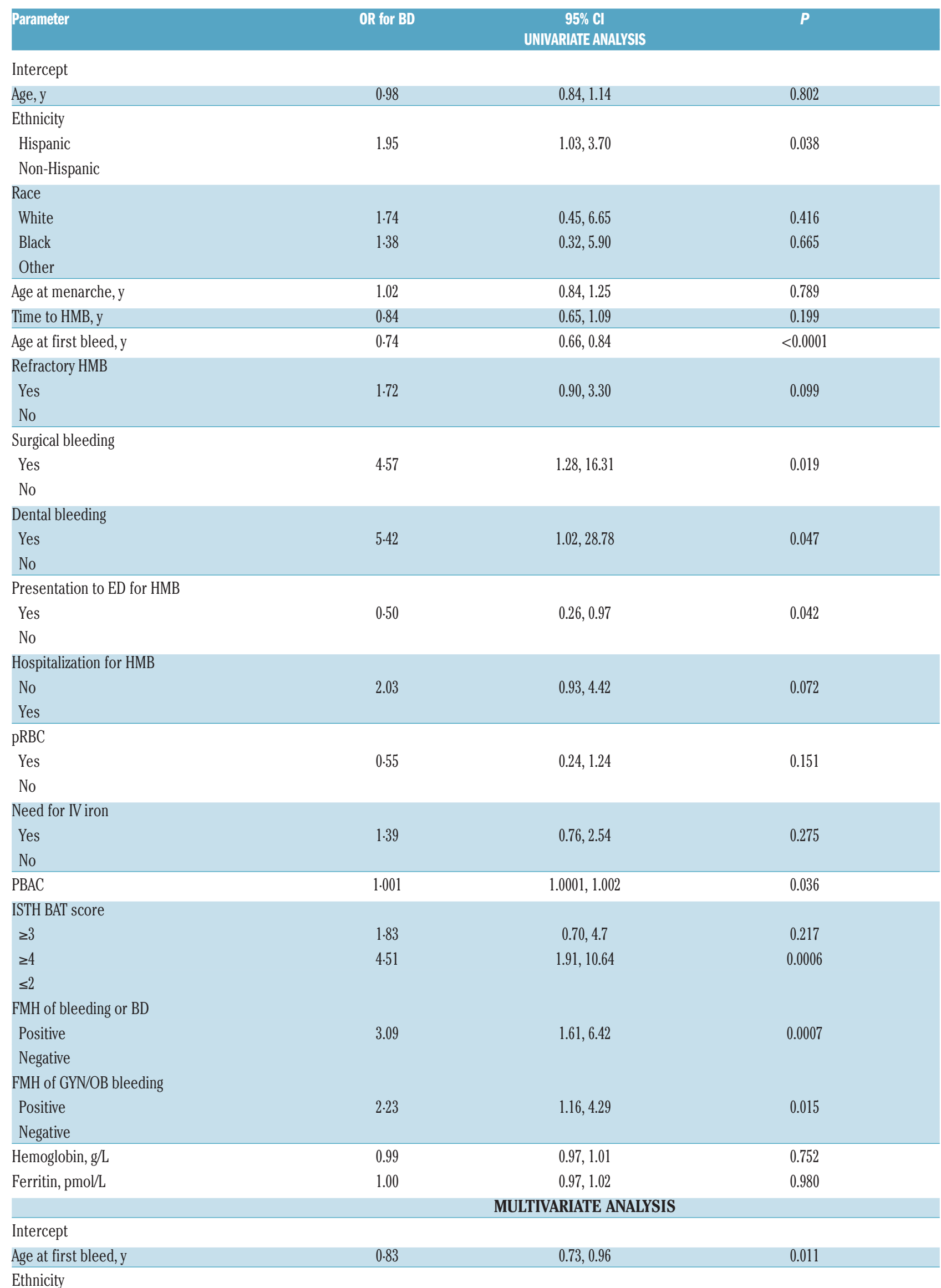

Ethnicity 
Hispanic

$2 \cdot 48$

Non-Hispanic

ED evaluation for HMB

Yes
No

ISTH BAT score

$\geq 3$

$\geq 4$
$0.05,0.38$

$<0.0001$

$\leq 2$

\section{C-statistic:}

$0.54,4.13$

$2.60,26.44$
0.021

BMI: body mass index; ED: emergency department; HMB: heavy menstrual bleeding; ISTH-BAT: International Society of Thrombosis Haemostasis-Bleeding Assessment Tool; pRBC: packed red blood cells; PBAC: pictorial blood assessment chart;TSH: thyroid-stimulating hormone; y: years.

those with BD had a diagnosis of either low vWF or vWD. Our findings suggest that $\mathrm{BD}$ are equally prevalent in the anovulatory or ovulatory pattern of menstrual bleeding. Identification of adolescents with $\mathrm{BD}$ is the first step in preventing delays in diagnosis and, by extension, longterm untoward complications of $\mathrm{BD}$. $\mathrm{HMB}$ soon after menarche is traditionally deemed as "hormonal," and anovulation is the default etiology. These findings have implications for clinicians who routinely manage adolescents with $\mathrm{HMB}$ : screening or referral for screening for $\mathrm{BD}$ is appropriate, irrespective of the pattern of menstrual bleeding.

Seravalli et al. investigated the frequency of $\mathrm{BD}$ in adolescents, dividing participants into two groups based on whether abnormal uterine bleeding started in the first two years from menarche or later. Overall, $48 \%$ of adolescents were diagnosed with a hemostatic defect (18\% with OPD, $14 \%$ with vWD, 13\% with clotting factor deficiencies, and $7 \%$ with an increase in bleeding time), but there were no differences in the prevalence of $\mathrm{BD}$ between the groups (44\% vs. $59 \%$; $P=0.17$ ). ${ }^{16}$ Philipp et al. also reported that adolescents and peri-menopausal women were just as likely to have hemostatic defects as were women aged 2044 years; ${ }^{17}$ the former age brackets representing periods of anovulatory menstrual bleeding. Furthermore, Vo et al. reported that adolescents with $\mathrm{BD}$ were more likely to perceive and report their menstrual cycles as irregular. ${ }^{18}$ These studies had certain limitations, such as retrospective data collection, and a lack of uniform and comprehensive laboratory investigation.

In our cohort, younger age at first bleeding event, Hispanic ethnicity, non-presentation to the ED for HMB, and ISTH BAT score of $\geq 4$ were identified as predictors of $\mathrm{BD}$. Younger age at first bleeding event is known to be associated with hemostatic evaluation and $\mathrm{BD}$ diagnosis, representing those with an earlier phenotypic expression or more severe bleeding phenotype. Hispanic girls in our cohort were more likely to have a $\mathrm{BD}$, even though nonHispanics made up the majority. Previous studies have included mostly non-Hispanics; more specifically, women of eastern European ancestry and a lower prevalence of vWD and higher levels of VWF antigen, vWF activity, and FVIII have been reported in black women, ${ }^{19,20}$ which can explain these findings. Adolescents who presented to ED for evaluation and management of $\mathrm{HMB}$ in our cohort were less likely to have a $\mathrm{BD}$, which is similar to results from another multicenter analysis. ${ }^{21}$ This may be further explained by: a) negative family history of $\mathrm{HMB}(60 \%$ of adolescents did not have a first-degree relative with $\mathrm{HMB}$ and the caregivers of these adolescents were likely unprepared to manage $\mathrm{HMB}$ at home); and b) $55 \%$ presenting to the ED had anovulatory bleeding (generally deemed difficult to manage with conventional route and doses of hormones). ${ }^{22}$ Moreover, $67 \%$ of those with anovulatory bleeding were overweight or obese, the latter being associated with gonadal steroid hormone changes that result in disruption of ovulation and menstrual irregularities including $\mathrm{HMB}{ }^{23,24}$ Previous studies show that adolescents who present acute symptoms and require hospitalization are more likely to have an underlying $\mathrm{BD}$ that was reported only in descriptive analyses, showing $19 \%$ and $33 \%$ of patients with abnormal uterine bleeding and coagulation disorders when compared with $74 \%$ and $67 \%$ without, respectively. $^{25-27}$

When objectively assessing bleeding in patients with $\mathrm{BD}$, consensus guidelines recommend the use of BAT. ${ }^{28}$ The ISTH BAT has been shown to optimally identify BD in both adults and children; the pediatric cut-off able to optimally discriminate between no $\mathrm{BD}$ and a possible $\mathrm{BD}$ is a score of $\geq 3 .{ }^{13}$ Instead, we identified a score of $\geq 4$ to be predictive of BD. Recently, using identical data, ISTH-BAT was shown to be more sensitive for assessment of $\mathrm{HMB}$ in women with low vWF levels compared with other BAT, supporting a need to investigate and validate a higher "adolescent" specific cut-off. ${ }^{29}$ The ISTH BAT score of $\geq 4$ in those with BD was driven mostly by the presence of additional bleeding symptoms with $\mathrm{HMB} ; 57 \%$ of adolescents with $\mathrm{BD}$ had other bleeding symptoms compared to $12 \%$ in those without BD. Previous data clearly show that the number of hemorrhagic symptoms is higher in the young when a more severe bleeding phenotype or disorder is present. ${ }^{30,31}$ The presence of at least three bleeding symptoms, irrespective of severity, result in $99.5 \%$ specificity for the most common bleeding disorder, vWD, a finding that has been confirmed in the pediatric age group. $^{32}$

The median time from onset of the first bleeding symptom to $\mathrm{BD}$ diagnosis was four years in the entire cohort, two years in the anovulatory, and six years in the ovulatory group. Adolescents with anovulatory $\mathrm{HMB}$ had heavier (mean PBAC score 427 vs. 345) or difficult to manage menses, resulting in an earlier referral and diagnosis, accounting for an earlier diagnosis. Lavin et al., on the other hand, have reported no differences in age at diagnosis for women with low vWF levels who reported HMB to physicians compared to those who did not go to the doctor (age 34.2 vs. 33.4 years; $P=0.7) .{ }^{29}$ Even though the time to diagnosis in our cohort is a significant improvement 
Table 4. Prevalence of non-hemostatic disorders and concomitant disorders.

\begin{tabular}{|c|c|c|c|c|}
\hline & $\begin{array}{c}\text { Anovulatory HMB } \\
\text { BD } \\
(n=31)\end{array}$ & $\begin{array}{l}\text { No BD } \\
(n=69)\end{array}$ & $\begin{array}{c}\text { Ovulatory HMB } \\
\text { BD } \\
(n=36)\end{array}$ & $\begin{array}{l}\text { No BD } \\
(n=64)\end{array}$ \\
\hline PCOS & 3 & 3 & 0 & 0 \\
\hline $\mathrm{BJH} \mathrm{H}^{* *}$ & 3 & 7 & 6 & 4 \\
\hline Uterine structural ab. & $1^{@}$ & $1^{\wedge}$ & $3^{@ @ ~}$ & 0 \\
\hline Systemic disorders & $6^{*}$ & $10^{!}$ & $1^{\#}$ & $4^{2 x}$ \\
\hline Exon 28 polym. & 0 & 1 & 0 & 3 \\
\hline
\end{tabular}

ab: abnormalities; BD: bleeding disorder; PCOS: polycystic ovarian syndrome; BJH: benign joint hypermobility; polym: polymorphism. **BJH assessment was performed only on 100 participants. Systemic or medical disorders: *depression $(n=4)$, remote history of cancer $(n=1)$, and hypothyroidism ( $\mathrm{n}=1)$; ! depression ( $\mathrm{n}=3$ ), asthma requiring medications ( $\mathrm{n}=3$ ), remote history of cancer ( $\mathrm{n}=3$ ), hypothyroidism ( $\mathrm{n}=1$ ); \#one had juvenile rheumatoid arthritis; ${ }^{*}$ depression ( $\mathrm{n}=1$ ), diabetes mellitus ( $\mathrm{n}=2$ ); celiac disease ( $\mathrm{n}=1$ ). Uterine structural abnormalities; ${ }^{\oplus}$ one had endometriosis; ^one had erosive vaginitis from tampon use; ${ }^{\oplus}$ two were diagnosed with endometriosis, and one was diagnosed with uterine polyps.

from the average delay of 16 years previously reported in women with $\mathrm{BD},{ }^{33}$ it highlights the importance of hematologists in the care of adolescents with HMB. For primary care practitioners, the relatively high prevalence of $\mathrm{vWD}$ in adolescent $\mathrm{HMB}$ is a reminder to test for $\mathrm{vWD}$. A recent retrospective analysis of a large national claims database of 23,888 post-pubertal girls and adolescents with $\mathrm{HMB}$ observed a very low rate of screening for vWD of only $8 \%$ in those with $\mathrm{HMB}$ and $16 \%$ in those with severe $\mathrm{HMB}^{34}$

The strengths of our study include the largest powered adolescent cohort ever prospectively examined. Participants underwent a uniform, protocolized evaluation for $\mathrm{BD}$ compared to "tiered" testing based on suspicion of a BD. Another finding of interest is an overall lower prevalence of OPD than that previously reported in the literature, where initial abnormal platelet aggregation was not repeated for confirmation. We diagnosed OPD based on reproducible findings, an approach that had been not undertaken in previous studies, leading to the overestimation of OPD (Online Supplementary Table S4 and Online Supplementary Figure S1).

Our study has some limitations. Although we consecutively enrolled unselected adolescents with HMB, they had, in effect, already been selected by their referring providers. This referral bias probably led to a higher frequency of $\mathrm{BD}$ in our center compared to primary care settings. This bias, however, is inherent to all studies carried out in tertiary care centers. The overall frequency of $\mathrm{BD}$ in our cohort is lower than that in previously reported data from other multidisciplinary clinics $(33 \%$ vs. $<60 \%)$ and is reflective of the stringent criteria used to diagnose $\mathrm{BD}$. We also excluded patients referred for abnormal coagulation profiles, including initial abnormal vWF analysis, which may have under-estimated the prevalence of $\mathrm{BD}$ in our cohort.
We did not exclude participants on hormonal suppression for HMB. Even though PBAC scores were calculated for pre-hormonal cycles, it may have impacted PBAC scores and its predictive ability due to recall bias. Moreover, PBAC has not been validated for retrospective use or without using standardized brand pads and tampons. Because our study was conducted in large academic centers with dedicated Young Women's Blood Disorders clinics, our results are unlikely to apply to settings with a low prevalence of $\mathrm{BD}$. Our time to diagnosis is subject to length bias; adolescents with severe bleeding phenotypes were likely referred earlier, leading to differences in time to diagnosis.

Our study will stimulate further research; the pediatric cut-off for an abnormal ISTH BAT score needs to be revisited for the adolescent age group. Even though we showed no difference in the frequency of $\mathrm{BD}$ according to HMB pattern, follow-up studies are in order on whether the efficacy of hemostatic-based therapies are equivalent. Finally, future studies are needed to assess the cost-effectiveness of selective testing in multidisciplinary clinics compared with universal screening across the population where the sole bleeding complaint could be HMB. Our predictive model also needs to be validated internally and externally in a larger population.

In summary, a high awareness, irrespective of the type of menstrual bleeding, is paramount to identify adolescents with $\mathrm{BD}$.

\section{Funding}

$A Z$ is supported by a grant from the National Institutes of Health (1K23HL132054-01). The funding source was not involved in the study design, analysis and interpretation of data; in the writing of the report or the decision to submit the article for publication.

\section{References}

1. Hallberg L, Hogdahl AM, Nilsson L, Rybo G. Menstrual blood loss--a population study. Variation at different ages and attempts to define normality. Acta Obstet Gynecol Scand. 1966;45(3):320-351.

2. Rosenfield RL. Clinical review: Adolescent anovulation: maturational mechanisms and implications. J Clin Endocrinol Metab. 2013;98(9):3572-3583.
3. Zia A, Rajpurkar M. Challenges of diagnosing and managing the adolescent with heavy menstrual bleeding. Thromb Res. 2016;143:91-100

4. Edlund $M$, Blomback $M$, von Schoultz B, Andersson $\mathrm{O}$. On the value of menorrhagia as a predictor for coagulation disorders. Am J Hematol. 1996;53(4):234-238.

5. Kadir RA, Economides DL, Sabin CA Owens D, Lee CA. Frequency of inherited bleeding disorders in women with menor- rhagia. Lancet. 1998;351(9101):485-489

6. Miller CH, Philipp CS, Stein SF, et al. The spectrum of haemostatic characteristics of women with unexplained menorrhagia. Haemophilia. 2011;17(1):e223-229.

7. Zia A Lau M, Journeycake J, et al. Developing a multidisciplinary Young Women's Blood Disorders Program: a singlecentre approach with guidance for other centres. Haemophilia. 2016; 22(2):199-207.

8. Munro MG, Critchley HOD, Fraser IS, 
Committee FMD. The two FIGO systems for normal and abnormal uterine bleeding symptoms and classification of causes of abnormal uterine bleeding in the reproductive years: 2018 revisions. Int J Gynaecol Obstet. 2018;143(3):393-408.

9. Philipp CS, Faiz A, Dowling NF, et al. Development of a screening tool for identifying women with menorrhagia for hemostatic evaluation. Am J Obstet Gynecol. 2008;198(2):163

10. ACOG Committee on Practice Bulletins-Gynecology. American College of Obstetricians and Gynecologists. ACOG practice bulletin: management of anovulatory bleeding. Int J Gynaecol Obstet. 2001;72(3):263-271.

11. Lang T, Bauters A, Braun SL, et al. Multicentre investigation on reference ranges for ROTEM thromboelastometry. Blood Coagul Fibrinolysis. 2005;16(4):301-310.

12. Higham JM, O'Brien PM, Shaw RW. Assessment of menstrual blood loss using a pictorial chart. Br J Obstet Gynaecol. 1990; 97(8):734-739.

13. Elbatarny M, Mollah S, Grabell J, et al. Normal range of bleeding scores for the ISTH-BAT: adult and pediatric data from the merging project. Haemophilia. 2014; 20(6):831-835

14. Liu XS. Implications of statistical power for confidence intervals. Br J Math Stat Psychol. 2012;65(3):427-437.

15. David W. Hosmer Jr. SL, Rodney X. Sturdivant. Applied Logistic Regression. 3rd Edition ed: John Wiley \& Sons. 2013.

16. Seravalli V, Linari S, Peruzzi E, Dei M, Paladino E, Bruni V. Prevalence of hemostatic disorders in adolescents with abnormal uterine bleeding. I Pediatr Adolesc Gynecol. 2013;26(5):285-289.
17. Philipp CS, Faiz A, Dowling N, et al. Age and the prevalence of bleeding disorders in women with menorrhagia. Obstet Gynecol. 2005;105(1):61-66.

18. Vo KT, Grooms L, Klima J, Holland-Hall C, O'Brien SH. Menstrual bleeding patterns and prevalence of bleeding disorders in a multidisciplinary adolescent haematology clinic. Haemophilia. 2013;19(1):71-75.

19. Kadir RA, Economides DL, Sabin CA Owens D, Lee CA. Variations in coagulation factors in women: effects of age, ethnicity, menstrual cycle and combined oral contraceptive. Thromb Haemost. 1999; 82(5):1456-1461.

20. Shankar M, Lee CA, Sabin CA, Economides DL, Kadir RA. von Willebrand disease in women with menorrhagia: a systematic review. BJOG. 2004;111(7):734-740.

21. Falcone T, Desjardins C, Bourque J, Granger L, Hemmings R, Quiros E. Dysfunctional uterine bleeding in adolescents. J Reprod Med. 1994;39(10):761-764.

22. Claessens EA, Cowell CA. Dysfunctional uterine bleeding in the adolescent. Pediatr Clin North Am. 1981;28(2):369-378.

23. Seif MW, Diamond K, Nickkho-Amiry M Obesity and menstrual disorders. Best Pract Res Clin Obstet Gynaecol. 2015;29(4):516527.

24. Pasquali R, Casimirri F, Plate L, Capelli M. Characterization of obese women with reduced sex hormone-binding globulin concentrations. Horm Metab Res. 1990; 22(5):303-306.

25. Claessens EA, Cowell CA. Acute adolescent menorrhagia. Am J Obstet Gynecol. 1981;139(3):277-280.

26. Smith YR, Quint EH, Hertzberg RB. Menorrhagia in adolescents requiring hospitalization. J Pediatr Adolesc Gynecol.
1998;11(1):13-15

27. Bevan JA, Maloney KW, Hillery CA, Gill JC, Montgomery RR, Scott JP. Bleeding disorders: A common cause of menorrhagia in adolescents. J Pediatr. 2001;138(6):856-861.

28. O'Brien SH. Bleeding scores: are they really useful? Hematology Am Soc Hematol Educ Program. 2012;2012:152-156.

29. Lavin M, Aguila S, Dalton $N$, et al. Significant gynecological bleeding in women with low von Willebrand factor levels. Blood Adv. 2018;2(14):1784-1791.

30. Elden L, Reinders M, Witmer C. Predictors of bleeding disorders in children with epistaxis: value of preoperative tests and clinical screening. Int J Pediatr Otorhinolaryngol. 2012;76(6):767-771

31. van Dijk K, Fischer K, van der Bom JG, Grobbee DE, van den Berg HM. Variability in clinical phenotype of severe haemophilia: the role of the first joint bleed. Haemophilia. 2005;11(5):438-443.

32. Bidlingmaier C, Grote V, Budde U, Olivieri $M$, Kurnik K. Prospective evaluation of a pediatric bleeding questionnaire and the ISTH bleeding assessment tool in children and parents in routine clinical practice. Thromb Haemost. 2012:10(7):1335-1341.

33. Kirtava A, Crudder S, Dilley A, Lally C, Evatt B. Trends in clinical management of women with von Willebrand disease: a survey of 75 women enrolled in haemophilia treatment centres in the United States. Haemophilia. 2004;10(2): 158-161.

34. Powers JM, Stanek JR, Srivaths L, Haamid FW, O'Brien SH. Hematologic considerations and Management of adolescent girls with heavy menstrual bleeding and anemia in US Children's Hospitals. J Pediat Adolesc Gynecol. 2018;31(5):446-450. 